\title{
Did synchronized ocean warming in the North Pacific and North Atlantic trigger a deglacial tipping point in the Northern Hemisphere?
}

Summer K. Praetorius' and Alan C. Mix²

\section{Rapid Northern Hemisphere warming during the last deglaciation involved synchronization of the North Pacific and North Atlantic. Threshold-like transitions to hypoxia occurred in conjunction with abrupt ocean warming, implying synergistic ocean heat transport triggered both physical and ecological tipping points.}

The rapid warming transitions into the Bølling-Allerød interstadial and Holocene interglacial are striking examples of nonlinearities in the climate system. A leading hypothesis for the trigger of these events has been changes in the strength of the Atlantic Meridional Overturning Circulation (AMOC) (McManus et al. 2004). However, records from the North Pacific and other distant locations document equally abrupt climate changes as those observed in the North Atlantic (Hendy and Kennett 1999; Wang et al. 2001; Praetorius and Mix 2014). This calls into question whether these are teleconnected responses to changes in the AMOC, or whether they involve orchestration of northern hemisphere climate dynamics and reflect critical transitions in the climate system, possibly involving multiple "tipping elements" (Lenton et al. 2008).

Proposed early warning signals (EWS) of tipping points include enhanced spatial correlation, increased autocorrelation, and high variance (Dakos et al. 2010; Scheffer et al.

2012). Evidence for enhanced variance and autocorrelation prior to the Bølling-Allerød and Holocene transitions is mixed, leading to debate as to whether these abrupt climate shifts are stochastic perturbations or climate bifurcations (Lenton et al. 2012). High spatial correlation may be a more reliable EWS (Dakos et al. 2010), but so far has not been widely applied to paleoclimate

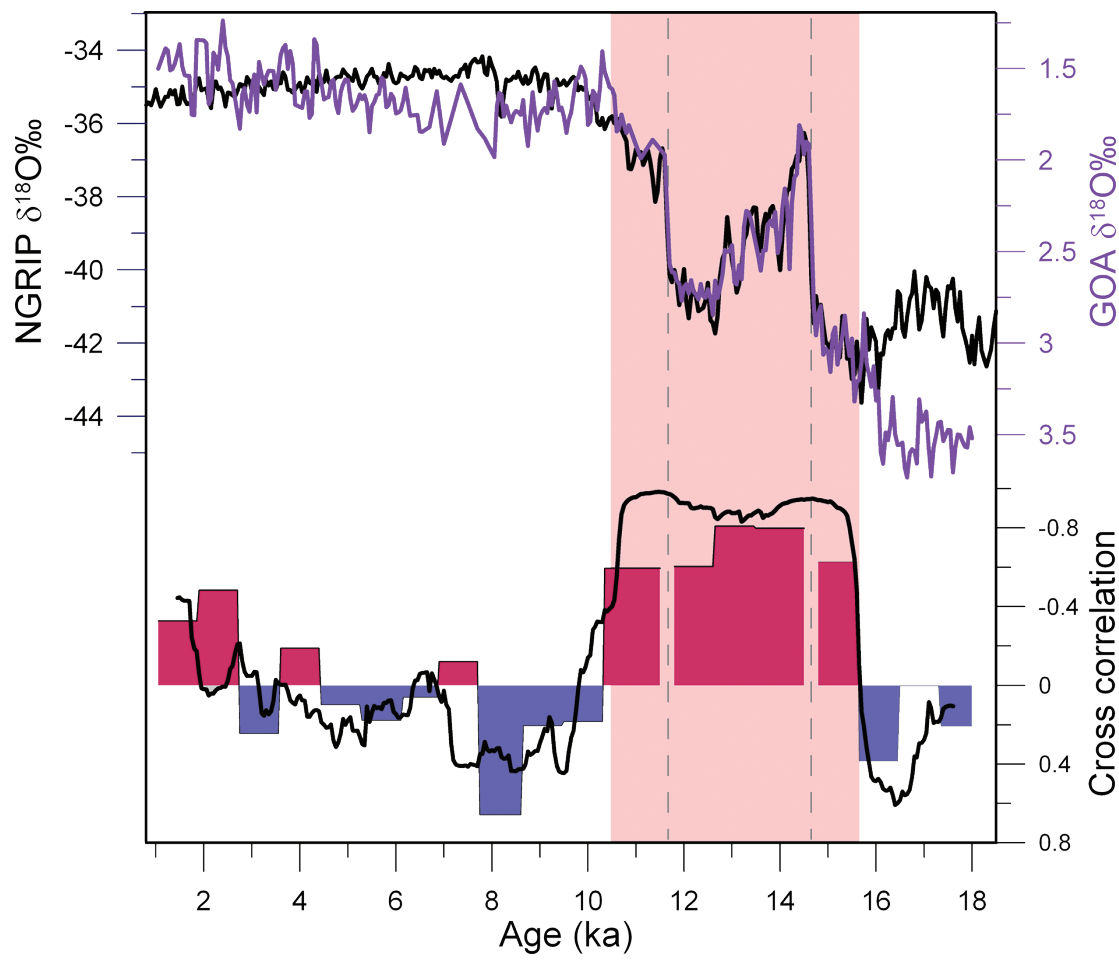

Figure 1: Changes in the correlation between the Greenland NGRIP (Rasmussen et al. 2006) and the Gulf of Alaska (GOA) $\delta^{18} \mathrm{O}$ records (inverted Y-axis; Praetorius and Mix 2014). Each record is interpolated on a $25 \mathrm{yr}$ time step with a $125 \mathrm{yr}$ Gaussian filter, and the cross correlation is evaluated with both a 2,000 yr centered moving average (black), and in 800 yr stationary windows (red: negative correlation, blue: positive correlation), excluding the 200 years surrounding the abrupt Bølling and Holocene transitions (dashed lines). Both the cross correlation in stationary windows and the moving average indicate a switch from positive to negative correlation (synchronized) prior to the Bølling transition. The interval of high negative correlation is highlighted with pink shading. Negative correlation implies a positive temperature relationship. A center-weighted moving average monitors broad trends in the record by approximating the timing of changes in cross correlation. It is not suited for detecting EWS in isolation of other evaluation as it smooths variance in abrupt transitions. Both the cross correlation in stationary windows and the moving average indicate a switch from positive to negative correlation (synchronized) prior to the Bølling transition. data. Detection of EWS in paleoclimate data remains challenging due to requirements for records with high signal-to-noise ratios and precise chronologies.

\section{North Pacific - North Atlantic climate flip-flop?}

We recently developed a decadal-resolution planktonic oxygen isotope record from the Gulf of Alaska with a centennial-scale radiocarbon chronology (Praetorius and Mix 2014) and compared changes to the Greenland NGRIP oxygen isotope record (Rasmussen et al. 2006). The two regions appear to flip-flop between correlation and anticorrelation (Fig. 1). A few hundred years prior to the abrupt warming transition into the Bølling-Allerød warm period at $14.7 \mathrm{ka}$ these records became synchronized, and maintained high correlation throughout the remainder of the deglaciation, encompassing abrupt climate fluctuations such as the Younger Dryas cooling episode and the rapid warming into the Holocene. Coupling of North Pacific and North Atlantic heat transport could act as an amplifying mechanism in abrupt northern hemisphere climate change, whereas opposing oceanic regimes could act to balance northern hemisphere heat transport, and thus promote climate stability.

Although this analysis was based on oxygen isotope data due to its high signal-to-noise ratio, $\delta^{18} \mathrm{O}$ may be sensitive to temperature, global ice volume changes, and local salinity effects. We have now expanded this work into specific sea-surface temperature proxies (Praetorius et al. 2015), and show that the rapid North Atlantic warming events (as recorded in Greenland) are indeed accompanied by abrupt, high-amplitude warming in the Northeast Pacific, and that abrupt changes in paleo-salinity play only a minor role in the oxygen isotope record. Because these massive warming events are found in the northward advective pathway of waters from lower latitudes, a substantial increase in net northward heat transport is likely. This is somewhat surprising, as the initial warming into the Bølling-Allerød occurred prior to the opening of Bering Strait, during a time when low salinity surface waters would have been trapped in the high North Pacific; radiocarbon evidence suggests that the warming events are not associated with enhanced local overturn (Davies et al. 2014). 
Coupled global climate models show an amplified surface warming in the North Pacific on centennial time scales in response to increasing radiative forcing due to a shallow mixed layer depth (Long et al. 2014). Such a mechanism may help to explain rapid North Pacific warming in concert with the deglacial rise in atmospheric $\mathrm{CO}_{2}$ concentrations.

\section{Ecological and biogeochemical} responses to physical tipping points

Biological and chemical systems may have been entrained in their own dynamics as the deglacial world tipped into warming. For example, it has been known for some time that the oxygen minimum zone expanded abruptly in the North Pacific during both the Bølling-Allerød and early Holocene (Jaccard and Galbraith 2012). These hypoxic events coincided with surface warming (Praetorius et al. 2015), implying strong feedbacks between ocean warming, deoxygenation, and marine productivity, with evidence for tipping-point impacts on the benthic fauna. Abrupt transitions to sedimentary laminations, in close association with enhanced burial of diatom algae, points to a strong role for enhanced sea-surface productivity and subsequent sinking of organic matter in pushing this system across a threshold of hypoxia that was sustained for millennia during each event. Sea-surface warming preceded the increase in productivity and initiation of hypoxia during the Bølling-Allerød transition, suggesting that warming triggered an array of biogeochemical feedbacks in the past, and may imply that future warming could trigger similar feedbacks, leading to more rapid or severe deoxygenation of the North Pacific than what is predicted based on thermal solubility alone.

An abrupt intensification of hypoxia is also observed in the Cariaco Basin in the tropical North Atlantic at similar times to those we observed in the North Pacific (Fig. 2; Gibson and Pederson 2014). The remarkably similar timing and magnitude of sea-surface temperature increase and the near synchronous onset of hypoxia in different oceanic regions in the North Pacific and North Atlantic during the Bølling-Allerød and Holocene transitions, in spite of rather different baseline conditions in the two oceans, imply a prominent role for ocean warming in pushing low-oxygen regions across thresholds of hypoxia. Exactly how the feedback mechanisms work remains poorly known and a subject for future observations and modeling.

\section{Outlook}

New high-resolution paleoceanographic records from the subpolar North Pacific document rapid changes during the last deglacial transition similar in timing to those observed in the Greenland ice cores. Rather than deglacial changes in the North Pacific merely reflecting a downstream response to changes in the North Atlantic region, interactions between basins may be a key element in the emergence of abrupt climate transitions in the Northern Hemisphere.

Although changes in inter-ocean coupling may have important consequences for

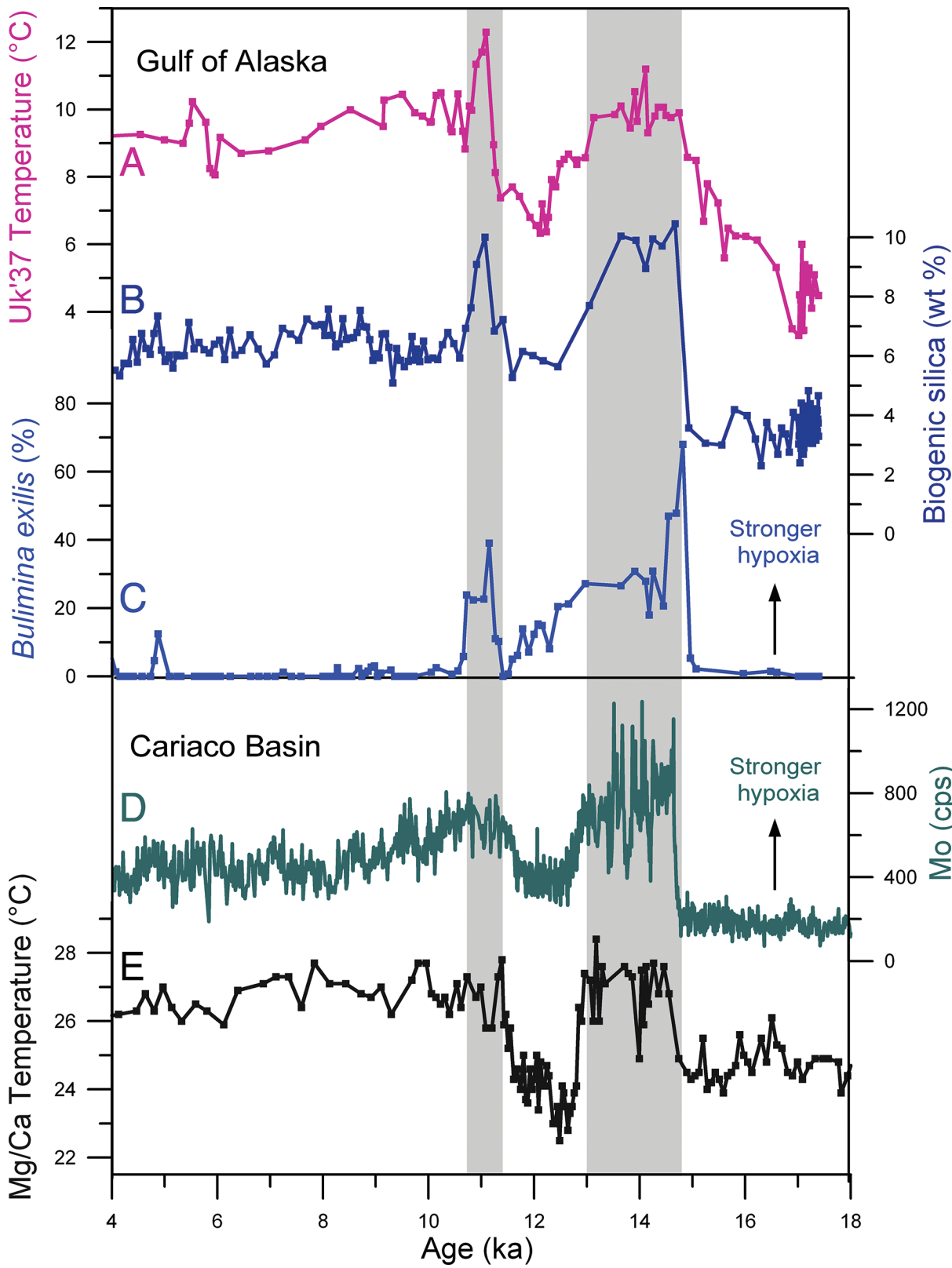

Figure 2: (A) Alkenone (Uk'37)-based sea surface temperature reconstructions from the Gulf of Alaska, North Pacific (Praetorius et al. 2015), with records of (B, C) productivity from the same location and (D) sedimentary redox and (E) temperature from the Cariaco Basin, North Atlantic. (B) Weight percent of biogenic silica (Davies et al. 2011) and (C) abundance of low-oxygen-tolerant benthic species Bulimina exilis (Praetorius et al. 2015). (D) Scanning-XRF record of Mo from the Cariaco Basin (Gibson and Pederson 2014), and (E) Mg/Ca paleotemperature reconstruction (Lea et al. 2003). Gray shaded bars reflect the laminated intervals in the Gulf of Alaska, which also correspond to intervals of strong hypoxia in the Cariaco Basin sediments.

climate, it remains unclear what regulates connectivity between the North Pacific and North Atlantic. This will be a worthy target for future studies. Development of high-fidelity paleoclimate records with precise and accurate chronology is challenging. Nevertheless, assessing the potential for tipping points in the future demands that we understand the dynamics of rapid climate changes and the biogeochemical responses and feedbacks they triggered in the past.

\section{AFFILIATIONS}

${ }^{1}$ Department of Global Ecology, Carnegie Institution for Science, Stanford, USA

${ }^{2}$ College of Earth, Ocean, \& Atmospheric Sciences, Oregon State University, Corvallis, USA

\section{CONTACT}

Summer Praetorius: spraetorius@carnegiescience.edu

\section{REFERENCES}

Dakos V et al. (2010) Theor Ecol 3: 163-174

Davies M et al. (2011) Paleoceanography 26 doi:10.1029/2010PA002051

Gibson K, Peterson L (2014) Geophys Res Lett 41: 969-975 Hendy I, Kennett J (1999) Geology 27: 291-294 Jaccard S, Galbraith E (2012) Nat Geosci 5: 151-156 Lea D et al. (2003) Science 301: 1361-1364 Lenton TM et al. (2008) PNAS 105: 1786-1793 Lenton TM et al. (2012) Clim Past 8: 1127-1139 Long S-M et al. (2014) J. Clim 27: 285-299 McManus J et al. (2004) Nature 428: 834-837

Praetorius S, Mix A (2014) Science 345: 444-448 Praetorius $S$ et al. (2015) Nature 527: 362-366 Rasmussen S et al. (2006) J Geophys Res 111, doi:10.1029/2005JD006079

Scheffer M et al. (2012) Science 338: 344-348 Wang Y et al. (2001) Science 294: 2345-2348 